\title{
Stereotaxic Magnetic Resonance Imaging Brain Atlases for Infants from 3 to 12 Months
}

\author{
Paul T. Fillmore ${ }^{a}$ John E. Richards ${ }^{b, c}$ Michelle C. Phillips-Meek ${ }^{b}$ Alison Cryer ${ }^{b}$ \\ Michael Stevens ${ }^{b, c}$ \\ Departments of a Communication Sciences and Disorders and ${ }^{b}$ Psychology, and ${ }^{\mathrm{C}}$ Institute for Mind and Brain, \\ University of South Carolina, Columbia, S.C., USA
}

\section{Key Words}

Atlas · Magnetic resonance imaging · Neurodevelopment · Infant

\begin{abstract}
Background: Accurate labeling of brain structures within an individual or group is a key issue in neuroimaging. Methods for labeling infant brains have depended on the labels done on adult brains or average magnetic resonance imaging (MRI) templates based on adult brains. However, the features of adult brains differ in several ways from infant brains, so the creation of a labeled stereotaxic atlas based on infants would be helpful. The current work builds on the recent creation of age-appropriate average MRI templates during the first year $(3,4.5,6,7.5,9$, and 12 months) by creating anatomical label sets for each template. Methods: We created stereotaxic atlases for the age-specific average MRI templates. Manual delineation of cortical and subcortical areas was done on the average templates based on infants during the first year. We also applied a procedure for automatic computation of macroanatomical atlases for individual infant participants using two manually segmented adult atlases (Hammers, LONI Probabilistic Brain Atlas-LPBA40). To evaluate our methods, we did manual delineation of several cortical areas on selected individuals from each age. Linear and nonlinear registration of the individual and average
\end{abstract}

template was used to transform the average atlas into the individual participant's space, and the average-transformed atlas was compared to the individual manually delineated brain areas. We also applied these methods to an external data set - not used in the atlas creation - to test generalizability of the atlases. Results: Age-appropriate manual atlases were the best fit to the individual manually delineated regions, with more error seen at greater age discrepancy. There was a close fit between the manually delineated and the automatically labeled regions for individual participants and for the age-appropriate template-based atlas transformed into participant space. There was close correspondence between automatic labeling of individual brain regions and those from the age-appropriate template. These relationships held even when tested on an external set of images. Conclusion: We have created age-appropriate labeled templates for use in the study of infant development at 6 ages $(3,4.5,6,7.5,9$, and 12 months). Comparison with manual methods was quite good. We developed three stereotaxic atlases (one manual, two automatic) for each infant age, which should allow more fine-grained analysis of brain structure for these populations than was previously possible with existing tools. The template-based atlases constructed in the current study are available online (http://jerlab.psych. sc.edu/NeurodevelopmentalMRIDatabase).

(c) 2015 S. Karger AG, Basel

\section{KARGER 125}

C 2015 S. Karger AG Basel

0378-5866/15/0376-0515\$39.50/0
John E. Richards

Department of Psychology

University of South Carolina

Columbia, SC 29208 (USA)

E-Mail richards-john@sc.edu 


\section{Introduction}

Magnetic resonance imaging (MRI) in infants allows for quantitative and qualitative assessment of neural development, which can enhance our understanding of early brain growth patterns and morphological changes during both normal and abnormal brain development. However, the quantitative study of infant brain development has been hampered by a lack of tools for structural analysis of the brain with MRI, in that tools which have been well validated in adults are often not validated for use with infants [1]. For example, adult neuroimaging work generally uses average MRI templates and stereotaxic atlases to identify anatomical locations in the brain, but these adult stereotaxic atlases may not identify locations accurately in the developing brain. In the current work, we created a set of anatomically labeled stereotaxic atlases for infants from 3 to 12 months (3, 4.5, 6, 7.5, 9, and 12 months). These atlases should be more accurate for infant participants than atlases created with adult participants.

There have been four primary approaches to neuroanatomical labeling of brain structures in adults, which are summarized in table 1 . All of these approaches result in a stereotaxic atlas, generally represented in an MRI volume, which identifies anatomical areas. The validity of these approaches may be tested against a 'gold standard' by manually delineating segmented regions in an individual participant and comparing an atlas which has been registered to the participant's brain against the manual delineation. The creation of the LONI Probabilistic Brain Atlas-LPBA40 [2], for example, required manual delineation of regions on the 40 subjects from whom the atlas was derived. This method is time consuming, typically requir- ing one or more individuals to be trained on manual delineation protocols. In addition, the results from one individual are not easily transferable to new MRIs. The development of methods that could make use of preexisting manual delineations would be highly desirable.

Studies of brain development in pediatric populations require similar atlases based on age-appropriate MRIs. One approach has been to register infant (or child, adolescent) brains to a labeled adult average template and transform the adult atlas back into the space of the developing brain. Recent studies have shown that the use of adult reference data compromises the analysis of infant brain images [3-5; for review, see 1]. There are at least two stereotaxic atlases that have been created for infants in the first 2 years $[6,7]$. Both of these atlases are based on registering infants to an atlas based on the manual segmentation of the average MRI of 1 young adult (A.A.L. [8]) and may remain biased by that individual's specific anatomy.

The current study constructed manually delineated and automatically derived anatomically labeled stereotaxic atlases for infant ages. Our goals were to construct the automatic atlases and validate them against manually delineated individual participant atlases. Thus, our first objective was to create a manually labeled, age-specific stereotaxic atlas for average MRI templates for infants at 3, 4.5, 6, 7.5, 9 , and 12 months of age on average MRI templates $[1,5,9$, 10]. We performed manual delineation of 14 areas (lobar and sublobar) on each infant-based age-specific template.

The second objective was to create automatically labeled atlases for infants in the first year by adapting the procedures used by Gousias et al. [11] with 2-year-olds. We used the 30 adults from the Hammers adult brain atlas (83 manually delineated areas; Hammers atlases [12-

Table 1. Approaches to neuroanatomical segmentation using stereotaxic atlases

\begin{tabular}{lll}
\hline Approach & Summary & Example \\
\hline Hand-drawn & $\begin{array}{l}\text { Anatomy hand drawn on the brains of a single or a few } \\
\text { participants and used as a guide; visual identification on new } \\
\text { individuals or look-up method }\end{array}$ & Talairach and Tournoux [31], 1988 \\
\hline Automatic-individual & $\begin{array}{l}\text { Atlases created by delineating regions on a single MRI volume; } \\
\text { individual images are then automatically registered to that image }\end{array}$ & $\begin{array}{l}\text { Automatic Anatomical Labeling } \\
\text { (Tzourio-Mazoyer et al. [8], 2002); Shi } \\
\text { et al. [7], 2011 }\end{array}$ \\
\hline Automatic-average & $\begin{array}{l}\text { Atlases created by delineating regions on the average of many MRI } \\
\text { volumes; individual images are then automatically registered to } \\
\text { that image }\end{array}$ & $\begin{array}{l}\text { MNI Structural Atlas (Mazziotta et al. } \\
\text { [32] 2001) }\end{array}$ \\
\hline Majority vote & $\begin{array}{l}\text { Manual delineation on a group of MRI volumes followed by fusion } \\
\text { of individual ones segmented with largest probability }\end{array}$ & $\begin{array}{l}\text { LONI Probabilistic Brain Atlas-LPBA40 } \\
\text { (Shattuck et al. [2], 2008); Gousias et al. } \\
\text { [11], 2008 }\end{array}$ \\
\hline
\end{tabular}


14]) and the 40 adults from the LONI Probabilistic Brain Atlas project (56 manually delineated areas; LPBA40 [2]) to create two macroanatomical atlases for individual infants at 3, 4.5, 6, 7.5, 9, and 12 months. The individual atlases from the infant participants were then used to create average age-specific macroanatomical atlases of 83 (Hammers) segments and 56 (LPBA40) segments, respectively.

The third objective was to examine the validity of the atlases. We tested the internal validity of the automatically generated macroanatomical atlases against each individual's manually segmented atlas, both for atlases created directly on the participant's brain and for the ageappropriate average atlas transformed into the participant space. To examine how atlas fit changed across age ranges, we also tested the individual participant's manually segmented brain regions against an 'age-appropriate' average MRI template and against templates based on ages older than the participants (from next oldest infant atlas through adult-based atlases). Finally, we examined external validity by testing the age-based template atlases against a new set of MRIs that were not used to create the atlases.

The template-based atlases constructed in the current study are available online (http://jerlab.psych.sc.edu/ NeurodevelopmentalMRIDatabase/) [10].

\section{Materials and Methods}

Participants, MRI Acquisition and Average MRI Templates

The participants, MRI acquisition and average MRI templates came from existing data sets, both from our laboratory and others $[5,10]$. The MRI images for the anatomical atlases came from the University of South Carolina McCausland Center for Brain Imaging (USC-MCBI). The averages were done at ages 3, 4.5 6, 7.5, 9, and 12 months and were based on 10-14 subjects at each age. The procedures for MRI acquisition are described elsewhere (USCMCBI $[5,10])$. The relevant MRI type for the current paper was a T1-weighted scan collected on a Siemens Medical System 3-tesla Trio with a 3D T1-weighted MPRAGE RF-spoiled rapid flash scan in the sagittal plane $\left(1-\mathrm{mm}^{3}\right.$ resolution, covering the entire head). The average templates were constructed on the MCBI 3-tesla T1weighted images with an iterative procedure $[9,15-17]$ and nonlinear registration methods (using ANTS, Advanced Normalization Tools $[18,19])$. The resulting average MRI template is an unbiased representation of the infant brain average. The average MRI templates are available online (http://jerlab.psych.sc.edu/Neurode velopmentalMRIDatabase/).

We also used MRI images for the external atlas validation from the NIH MRI Study of Normal Brain Development (NIHPD). The NIHPD MRI images were from the Objective-2 sample and were selected for infants who were within \pm 2 weeks of ages 3 months $(\mathrm{n}=21,10$ female, $\mathrm{F} / 11$ male, $M), 6$ months $(\mathrm{n}=32,15 \mathrm{~F} / 17 \mathrm{M})$, 9 months $(n=29,16 \mathrm{~F} / 13 \mathrm{M})$, or 12 months $(n=25,11 \mathrm{~F} / 14 \mathrm{M})$.
Detailed methodology for the NIHPD Objective- 2 can be found in publications [20, 21], as well as from the Objective-2 Procedure Manuals available at the NIHPD Database Repository website (https://nihpd.crbs.ucsd.edu/nihpd/info/index.html). The NIHPD scans were collected with Siemens Medical Systems (Sonata, Magenatom) and GE Systems (Signa Excite) 1.5-tesla scanners at two different sites and were a $2 \mathrm{D}$ scan in the axial plane $(1 \times$ $1 \times 3 \mathrm{~mm}$ resolution).

\section{Anatomically Labeled Stereotaxic Atlas Creation}

Four sets of atlases were created (table 2). These included the following: (1) manually delineated atlases for each of the average 3-tesla MRI templates at ages 3, 4.5, 6, 7.5, 9, and 12 months, (2) manually delineated atlases for 20 individual participants (used for internal and external validity tests), (3) automated macroanatomical atlases for all MCBI and NIHPD participants and (4) automated macroanatomical atlases for the 3-tesla MCBI average templates.

Manually Delineated Atlases: Average Templates. The manually drawn template atlases were constructed by manual delineation of 14 areas on each average MRI template. Adaptations of the LONI manual segmenting protocol was used to define the lobar areas (frontal, temporal, parietal, occipital, and insular), sublobar areas (fusiform, cingulate) and subcortical areas (cerebellum, brainstem, thalamus, corpus callosum, and striatum [2]), as well as the ventricles. Our manual delineation sequence is listed in table 3. The resulting areas were put in a single MRI volume, with the sublobar areas masking out the corresponding voxels from the lobar areas; we also retained each area in a separate MRI volume. An atlas was constructed for each of the 3-tesla average MRI templates for the infant ages $(3,4.5,6,7.5,9$, and 12 months). The atlases were delineated by one individual and compared against the delineation of a second observer for 6 areas (brainstem, cerebellum, thalamus, frontal, temporal, occipital). Over the 6 infant ages and 6 brain areas the overlap of the final average template atlas and the test observer was 0.973 and the median Dice agreement between the two observers was 0.958 (range $=0.89-0.99$ ).

Manually Delineated Atlases: Individual Participants. Selected participants had manual delineation done for 3 noncortical areas (cerebellum, brainstem and thalamus) and 3 cortical lobes (frontal, temporal and occipital). The delineation for the individuals was done in the same manner as the average templates. In detail, 2 of the participants at each infant age from the MCBI MRIs were randomly chosen as test participants for manual delineation and 2 participants from the NIHPD MRIs at 3, 6, 9, and 12 months were also chosen for manual delineation. Thus, there were 4 manually delineated participants at $3,6,9$, and 12 months and 2 manually delineated participants at 4.5 and 7.5 months of age. The manual delineations were done by several individuals and compared against the delineation of a single observer for 6 areas (brainstem, cerebellum, thalamus, frontal, temporal, occipital). Over the 20 participants and 6 brain areas the overlap of the final average template atlas and the test observer was 0.943 and the median Dice agreement between the two observers was 0.880 (range $=0.84-0.93$ ).

Automated Macroanatomical Atlases: Individual Participants. Four atlases were constructed on the MRIs of the individual participants based on 2 independent sets of labeled brain images and 2 registration types. The adult brain segmentations from www. brain-development.org (Hammers atlases [12, 22, 23]) were used as one atlas and the LONI Probabilistic Brain Atlas segmentations (40 individuals, 56 manually delineated areas; LPBA40 [2]) were 
Table 2. The four types of atlases

Average template manually delineated atlas

Manual delineation of 14 lobar and sublobar areas for each average MRI template (3, 4.5, 6, 7.5, 9, and 12 months); this is an average atlas that may be used for automatic atlas registration/transformation to individual participants (table 1, automatic-average)

Participant manually delineated atlas

Manual delineation of 14 lobar and sublobar areas for 20 selected participants (2 MCBI 3-tesla MRIs each at 3, 4.5, 5, 7.5, 9, and 12 months; 2 NIHPD 1.5 -tesla MRIs each at $3,6,9$, and 12 months); this is to provide a 'gold standard' against which to validate the average template manually delineated atlas and the automatically generated atlases (table 1 , hand-drawn)

Participant macroanatomical atlas

Automatic creation of macroanatomical atlas based on Hammers and LPBA40 atlases, using the method of registration of Gousias et al. [11] and majority vote; this was done for all MCBI participants making up the average MRI templates and all NIHPD participants at ages 3, 6, 9, and 12 months; linear: linear registration of the segmented adult atlases to the individual infant participants; nonlinear: nonlinear registration of the segmented adult atlases to individual infant participants; these atlases represent an approach to construct a macroanatomical atlas for individual infant participants (table 1, majority vote)

Average template macroanatomical atlases

Automatic creation of macroanatomical atlas based on Hammers and LPBA40 atlases; based on majority vote procedure with individual MCBI participant macroanatomical atlases making up the average MRI templates; this was done at 3, 4.5 6, 7.5, 9, and 12 months; linear: linear registration of the linear-constructed participant macroanatomical atlas to the average MRI template; nonlinear: nonlinear registration of the nonlinear-constructed participant macroanatomical atlas to the average MRI template; this is an average atlas that may be used for automatic atlas registration/transformation to individual participants (table 1, automatic-average)

Table 3. Steps for delineation of manual atlas

(1) Mask the insula and noncortical areas; noncortical areas include cerebellum, brainstem, thalamus, corpus callosum, and striatum (putamen, caudate); use the protocols developed for the LPBA40 atlas

http://resource.loni.usc.edu/resources/downloads/research-protocols/probalistic-atlas/cerebellum/

http://resource.loni.usc.edu/resources/downloads/research-protocols/probalistic-atlas/brainstem/

http://resource.loni.usc.edu/resources/downloads/research-protocols/masking-regions/thalamus/

http://resource.loni.usc.edu/resources/downloads/research-protocols/segmentation/caudate-delineation/

http://resource.loni.usc.edu/resources/downloads/research-protocols/masking-regions/insular-cortex/

(2) Identify the ventricles using an average T2-weighted MRI; thresholding of the CSF by using the values found in the internal ventricles; use this threshold to identify CSF in ventricles throughout brain

(3) Mask the frontal and temporal lobes; use the LPBA40 protocols to identify the areas for either frontal or temporal lobes http://resource.loni.usc.edu/resources/downloads/research-protocols/masking-regions/frontal-lobe-left/

Temporal:

http://resource.loni.usc.edu/resources/downloads/research-protocols/probalistic-atlas/inferior-temporal-gyrus/

http://resource.loni.usc.edu/resources/downloads/research-protocols/probalistic-atlas/parahippocampal-gyrus/

http://resource.loni.usc.edu/resources/downloads/research-protocols/probalistic-atlas/middle-temporal-gryus/

http://resource.loni.usc.edu/resources/downloads/research-protocols/masking-regions/middle-temporal-gyrus/

http://resource.loni.usc.edu/resources/downloads/research-protocols/masking-regions/superior-temporal-gyrus/

http://resource.loni.usc.edu/resources/downloads/research-protocols/probalistic-atlas/superior-temporal-gyrus/

(4) Mask the occipital area; for occipital area, use the MNI Structural Atlas definition of Mazziotta et al. [32] provided with FSL; a first approximation was generated by registering the infant average template to the 20 - to 24 -year template and transforming the lobar atlas from this template to the infant average template; the areas were then manually adjusted for the infant atlas

(5) Mask the cortical sublobar areas (fusiform, cingulate); the definition from the FSL Harvard-Oxford Atlases (Desikan et al. [33], 2006) and the LONI protocols were used; a first approximation was generated by registering the infant average template to the 20- to 24-year template and transforming the lobar atlas from this template to the infant average template; these areas were manually adjusted to match the areas for the infant atlas and adjusted to match the areas in the LONI segmenting protocols

http://resource.loni.usc.edu/resources/downloads/research-protocols/probalistic-atlas/fusiform-gyrus/

http://resource.loni.usc.edu/resources/downloads/research-protocols/probalistic-atlas/cingulate-gyrus/

(6) Mask the parietal lobe; the parietal lobe was the brain area in the parietal area left after the surrounding areas were identified (e.g. occipital, frontal, temporal) and was manually adjusted to match the separate areas in the LONI segmenting protocols

http://resource.loni.usc.edu/resources/downloads/research-protocols/masking-regions/parietal-lobe/

(7) The resulting areas were put in a single MRI volume, with the sublobar areas (cingulate, fusiform gyrus) masking out the corresponding voxels from the lobar areas; all 14 areas were retained in separate MRI volumes 
used for the other atlas. Each individual infant's MRI-extracted brain was registered (both linearly and nonlinearly) to each of the adult brains (both Hammers and LPBA40) and each labeled adult atlas was transformed to the individual infant space. These labeled sets (e.g. 30 individuals, for Hammers atlas) of linear-transformed atlases were then fused in a majority vote procedure $[7,11]$. The resulting atlas identifies the majority-voted brain segment for each voxel of the individual infant brain. The same procedure was done separately for both sets of labeled atlases. Therefore, each infant participant had a linear Hammers atlas, a nonlinear Hammers atlas, a linear LPBA40 atlas, and a nonlinear LPBA40 atlas.

Automated Macroanatomical Atlases: Average Templates. The Hammers and LPBA40 labeled atlases were constructed for the average MRI template from a majority vote procedure, using the individual participants from whom the average was made. For example, for the 3-month average template, each of the $14 \mathrm{MCBI}$ infants at 3 months of age were registered (both linearly and nonlinearly) to the 3-month-old average template and the majority vote fusion procedure was used to construct both Hammers and LPBA40 atlases. The Hammers and LPBA40 atlases for each participant were then transformed separately by both linear and nonlinear registration parameters to the average MRI template space and the resulting individually transformed atlases were fused with the majority vote procedure $[7,11]$ to construct the labeled average MRI template stereotaxic atlas. Thus, as with the individual participants, each average MRI template had a linear Hammers atlas, a nonlinear Hammers atlas, a linear LPBA40 atlas, and a nonlinear LPBA40 atlas. The NIHPD participants at 3, 6, 9, and 12 months were not used to create these averages and were reserved for an external validation of the atlases.

\section{Linear and Nonlinear Registration}

Linear and nonlinear registration methods were used in the construction of the atlases and in our evaluation tests. For linear registration, the FMRIB's Linear Image Registration Tool from FSL (FLIRT [24]) was used to register the MRI brain volume of the participant to a reference brain volume (infant participant to segmented adult atlases for participant macroanatomical atlas; infant participant to average MRI template for average template macroanatomical atlas). The FLIRT procedure was done with a 12-DOF affine search, and 'cost' and 'searchcost' used the 'corratio' algorithm. The linear registration produces a linear affine transformation matrix that can be applied to MRI volumes in the participant's space (e.g. individual participant atlas) to transform the volume to the reference space, and an inverse transformation matrix can be applied to MRI volumes in the reference space (e.g. adult manually segmented atlas) to transform the volume into the participant space. The ANTS was used for nonlinear registration [18]; ANTS version 2.1.0 was used, with the ANTS registration procedure and the cross-correlation metric, to a $1-\mathrm{mm}$ voxel resolution and the SyN transform (gradient step 0.1).

\section{Results}

Several individual and average atlases were successfully created. Manually delineated template-based atlases were created for each target infant age $(3,4.5,6,7.5,9$, and 12 months). Figure 1 shows the manual template on the 6 infant ages (fig. 1a sagittal; fig. $1 \mathrm{~b}$ axial), along with the manual template for the atlas of the young adults. Figure $2 \mathrm{a}$ shows $3 \mathrm{D}$ color rendering of the manual atlas overlaid on the average MRI template for each infant age and the young adults. For selected participants at each age, the 6 manually delineated brain areas were done (cerebellum, brainstem, thalamus, frontal lobe, temporal lobe, occipital lobe). Figure $2 \mathrm{~b}$ shows a 3D color rendering of these 6 areas on a 3-month-old participant. The Hammers and LPBA40 macroanatomical atlases were done for all individuals (MCBI and NIHPD). Figure 3a shows the Hammers and LPBA40 atlases on a 3-month-old and a 12 -month-old individual. Figure $3 \mathrm{~b}$ shows the Hammers and LPBA40 atlases on the MRI templates for the 3 -month-old and 12-month-old infants. Figure 4 shows axial slices of the average MRI template for the 6-monthold participants, along with slices at the same level for the manual atlas, Hammers atlas and the LPBA40 atlas. The template-based atlases constructed in the current study are available online (http://jerlab.psych.sc.edu/ NeurodevelopmentalMRIDatabase/) [10]. This includes the manual lobar atlas, the LPBA 40 atlas and the Hammers atlas for each age-appropriate average MRI template.

The overlap of the manually drawn atlas and the Hammers and LPBA40 atlases were examined for infant atlases to evaluate the overlap of the manual and the two automatically generated atlases. The overlaps between the areas of the manual atlas and the Hammers and LPBA40 atlases are given in table 4 . We used the overlapping areas found in table 4 to recode the individual participant Hammers or LPBA40 atlases into comparable manual atlas areas for some of the analyses. For these analyses, we did not examine the manually defined occipital lobe because of the disparity between our definition and corresponding LPBA40/Hammers atlases. Additionally, the LPBA40 atlas did not delineate the thalamus.

\section{Manually Delineated Regions versus Registration/} Transformation to Template Atlases of Various Ages (Table 5, Comparison 1)

Table 5 is a summary of our comparisons. The first analysis examined the effect of age-appropriate atlases by looking at the overlap between the manually delineated region of an individual, with the regions resulting from registration/transformation to the age-appropriate atlas and to all older age template-based atlases (all infant ages, 2 years, 12 years, 18 years, 20-24 years). This was done for the participants with manually delineated regions. The line graphs in figure 5 show the mean Dice value as a func- 


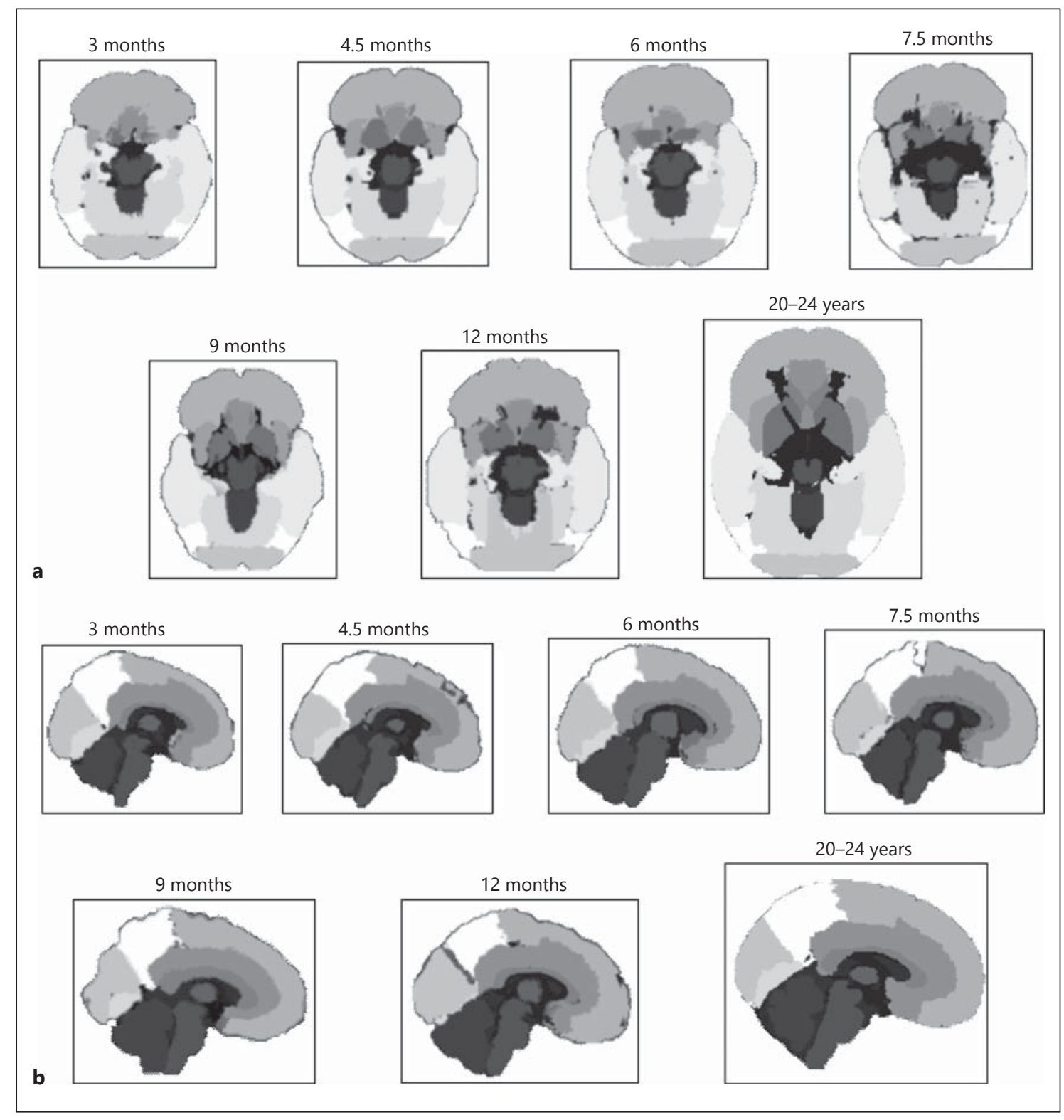

Fig. 1. Manual template-based atlas for 6 ages through the first year and adult atlas. a Axial view. b Sagittal view.

tion of the age of the template-based atlas separately for each age group. The registration/transformation for fig. 5 a was the linear registration, with the nonlinear registration shown in fig. 5b. Each subpanel shows the results for 1 of 6 different brain regions. In almost all cases the age-appropriate registration/transformation areas provided the largest overlap with the manually delineated regions of an individual. There was a gradual decrease on the Dice overlap as the difference between the participant age and the template age increased. The analogous results for the nonlinear registrations were similar for all areas except the brainstem and thalamus. The nonlinear registrations seemed to eliminate the age effects for these segments.

We compared the fit of the age-appropriate infant atlas, the atlas from the 2-year-old children and the adult atlas. The bar graph in figure 6 shows the mean Dice value as a function of the brain region with a bar for each age of the template-based atlas (same age as participant, 2 years or 20-24 years) for both linear and nonlinear registrations. The Dice value was analyzed with an age (3 ages: age-appropriate, 2 years, $20-24$ years) $\times$ registration 
Fig. 2. a Manual 3D template-based atlases for 6 ages through the first year and adult atlas; presented at the midsagittal plane. b Example of manual delineations on a 3-month-old infant brain (left $=$ medial view; right $=$ lateral view).

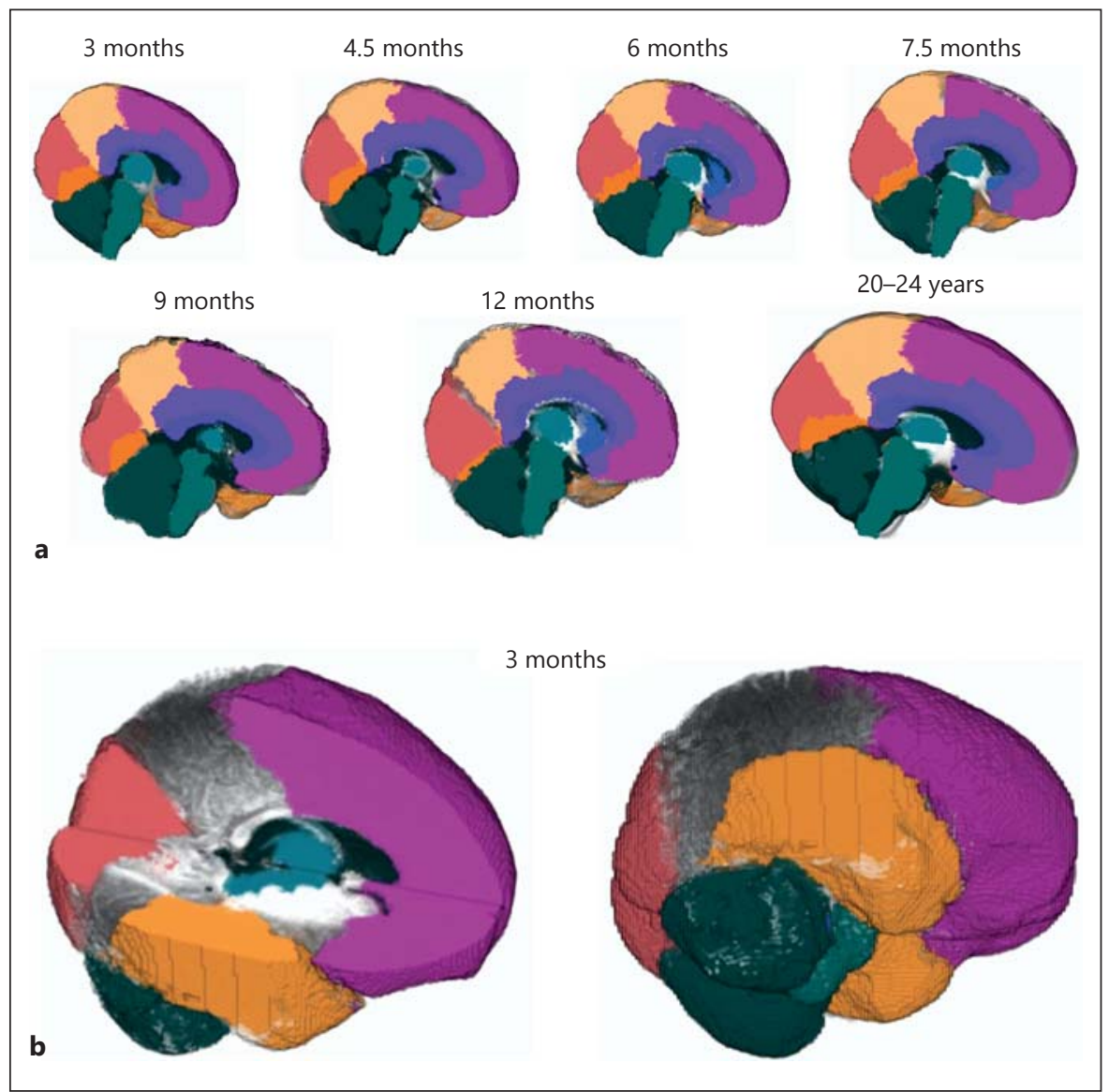

type (linear, nonlinear) $\times$ segment (cerebellum, brainstem, thalamus, frontal lobe, temporal lobe, occipital lobe) ANOVA. There was a significant main effect of age $\left(\mathrm{F}_{2,38}=131.24, \mathrm{p}<0.0001\right)$, such that overlap was higher between the manually delineated regions and registration/transformation to the age-appropriate atlas (mean = 0.851 , standard error of the mean, $\mathrm{SE}=0.0022$ ) than registration/transformation to either the 2-year-old (mean = $0.812, \mathrm{SE}=0.0028)$ or 20 - to 24 -year-old (mean $=0.809$, $\mathrm{SE}=0.0026)$ atlases. There was also a significant main effect of segment $\left(\mathrm{F}_{5,95}=40.81, \mathrm{p}<0.0001\right)$. There was no main effect of registration type but there was a significant 3-way interaction between age, registration type and segment $\left(\mathrm{F}_{10,190}=27.61, \mathrm{p}<0.0001\right)$. We used the Scheffé post hoc correction procedure to examine this interaction separately for linear and nonlinear registrations. For linear registration, the age effect was significant (e.g. ageappropriate templates performed better than those for older ages) for all 6 brain areas (fig. 6a). For nonlinear registration, the cerebellum and frontal, occipital and temporal lobes showed significant age effects, whereas the age effect for the brainstem and thalamus comparisons was not significant (fig. 6b). The nonlinear transform resulted in larger Dice values for the thalamus and brainstem segments.

\section{Manually Delineated Regions versus Individual and Average Macroanatomical Atlases (Table 5, Comparison 2)}

The second analysis was to examine the overlap between the manually delineated region for each individual, with the regions from that individual's Hammers and LPBA40 atlases, and with the regions from an age-appropriate MRI template atlas transformed into that participant's MRI space. This was done for the participants with manually delineated regions. The Hammers and LPBA40 atlas segments were recoded into regions equivalent to the manual atlas areas in order to compare the regions defined by the manual atlas with comparable regions defined by the average template macroanatomical atlases. This was accomplished by using any atlas segment where the overlap between the manual atlas and the target atlas 


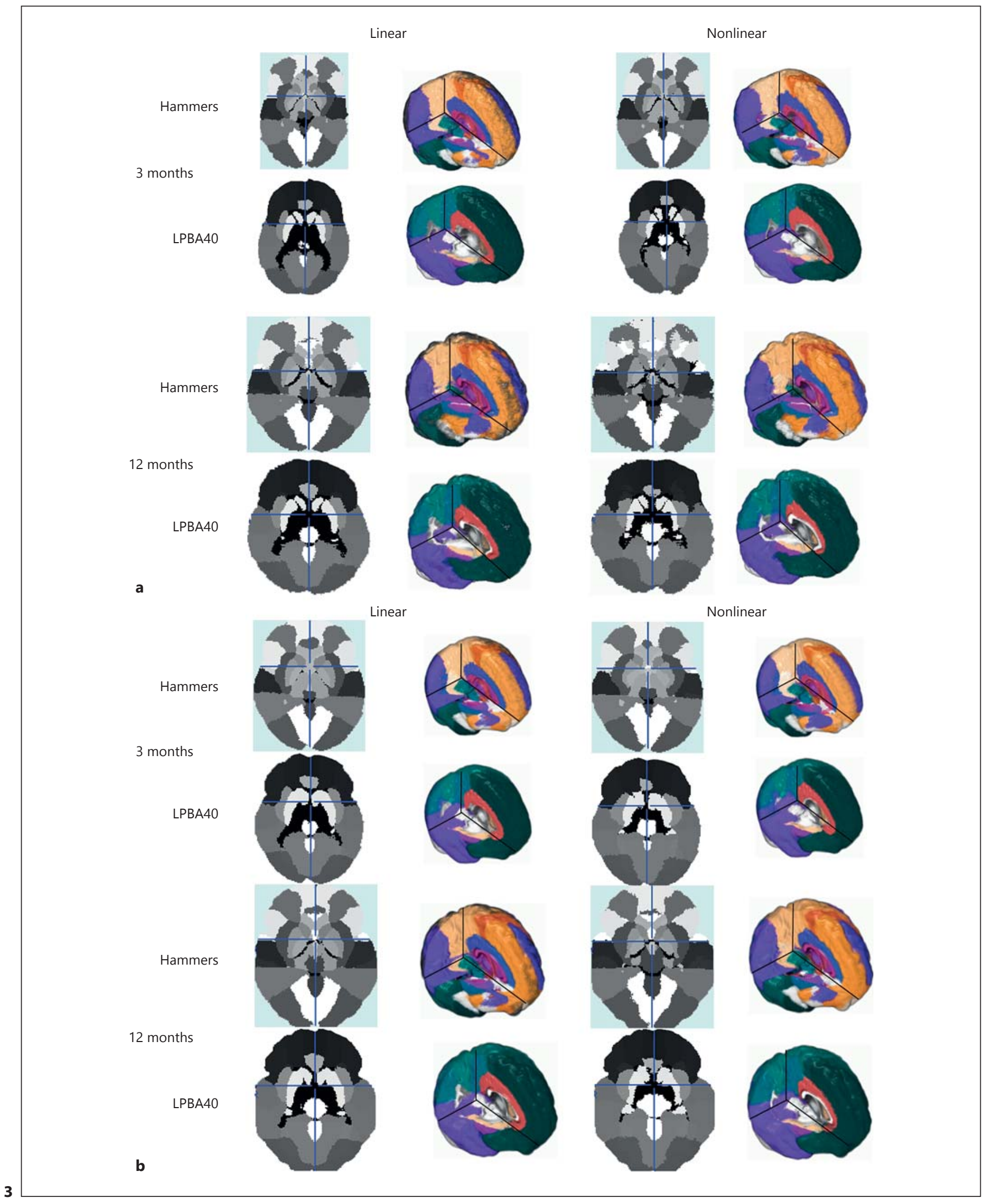

(For legend see next page.) 
area was greater than 0.5 (table 4). The first ANOVA used data provided by the Hammers atlases. The Dice value was analyzed with an age (6 ages: 3, 4.5, 6, 7.5, 9, 12 months) $\times$ segment ( 5 segments: cerebellum, brainstem, thalamus, frontal lobe, temporal lobe) $\times$ registration type (linear, nonlinear) $\times$ atlas source (participant, averagetransformed) ANOVA. There were significant effects of the registration type $\left(\mathrm{F}_{1,14}=144.8, \mathrm{p}<0.0001\right)$ and atlas source $\left(\mathrm{F}_{1,14}=15.82 \mathrm{p}=0.0014\right)$. The Dice coefficients from the linear analysis were larger than the nonlinear $(\mathrm{n}=200$, mean $=0.843$ and $0.782, \mathrm{SE}=0.0043$ and 0.0058 , respectively) and the Dice coefficients from the individual participant atlas were larger than those from the agetransformed atlas $(n=200$, mean $=0.809$ and $0.779, S E=$ 0.0048 and 0.0053 , respectively). It is interesting that the interaction between these two factors was not statistically significant, indicating that the linear/nonlinear change in the Dice coefficient was the same for the age-appropriate average-transformed comparison and the individual macroanatomical atlas comparison. For the ANOVA on the LPBA40 data, there were also significant effects of the registration type $\left(\mathrm{F}_{1,15}=78.84, \mathrm{p}<0.0001\right)$ and atlas

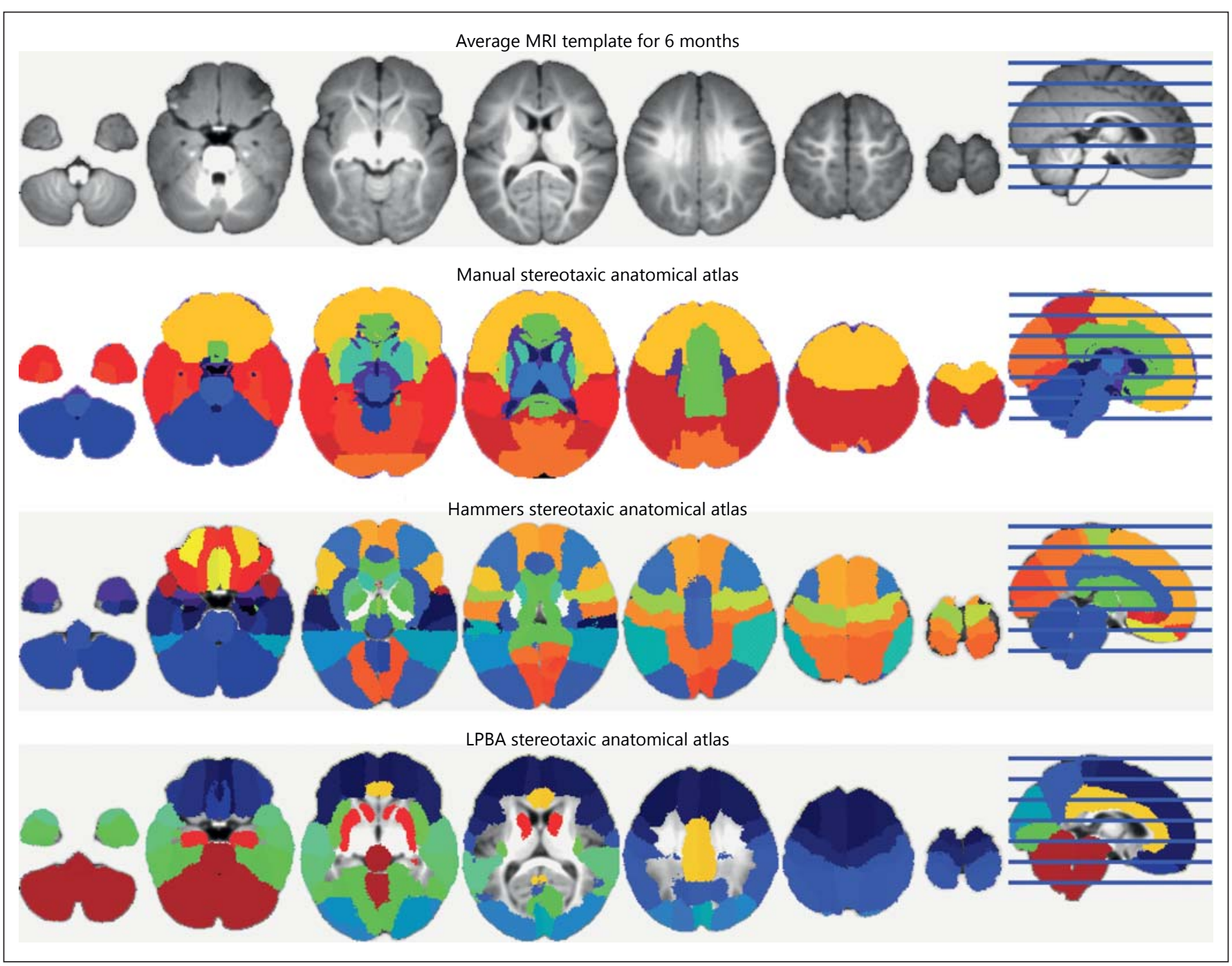

Fig. 4. Slices of average MRI template for 6-month-old participants compared with manual, Hammers and LPBA40 atlases.

Fig. 3. Hammers and LPBA40 atlases. Axial view is shown in black and white with AC-PC line; sagittal/medial view is shown in color (color in online version only). a A 3-month-old and a 12-month-old resulting from the individual majority vote procedure. b Age-appropriate average atlases shown on the 3 -month and 12-month average templates. 
Table 4. Overlap between the areas of the manual atlas and the LPBA40 and Hammers atlases




Table 4. (continued)

\begin{tabular}{|c|c|c|c|c|c|c|}
\hline \multirow{2}{*}{$\frac{\text { Manual atlas label }}{14 \text { Parietal }}$} & \multicolumn{2}{|c|}{ Hammers atlas label } & \multirow{2}{*}{$\begin{array}{l}\% \text { overlap } \\
80,78\end{array}$} & \multicolumn{2}{|c|}{ LPBA40 atlas label } & \multirow{2}{*}{$\begin{array}{l}\text { \% overlap } \\
84,85\end{array}$} \\
\hline & 60,61 & Postcentral gyrus (left, right) & & 41,42 & Postcentral gyrus (left, right) & \\
\hline & 62,63 & Superior parietal gyrus (left, right) & 81,80 & 43,44 & Superior parietal gyrus (left, right) & 84,84 \\
\hline & 32,33 & Remainder of the parietal lobe (left, right) & 89,91 & 45,46 & Supramarginal gyrus (left, right) & 82,88 \\
\hline & 22,23 & Lateral remainder of occipital lobe (left, right) & 45,45 & 47,48 & Angular gyrus (left, right) & 86,85 \\
\hline & & & & 49,50 & Precuneus (left, right) & 80,77 \\
\hline & & & & 63,64 & Middle occipital gyrus (left, right) & 46,45 \\
\hline & & & & 65,66 & Inferior occipital gyrus (left, right) & 22,35 \\
\hline
\end{tabular}

Numbers are the segment numbers on the atlas. \% overlap is the shared overlap between the manual atlas and the other atlases averaged across the 6 infant ages.

Table 5. Comparisons between atlas approaches examined in the current study

(1) Manually delineated regions vs. registration/transformation to template atlas Overlap between manually delineated regions and regions resulting from linear/nonlinear registration/transformation to the age-appropriate template-based atlas and older age template-based atlases; the Dice coefficient (Dice [34], 1945; Crum et al. [26], 2005) was used to evaluate the overlap of MRI atlas volumes; the Dice coefficient measures degree of overlap (ranging from 0 , no overlap to 1 , total overlap) and represents the intersection of 2 similarly labeled regions divided by the mean volume of the regions. We also calculated the 'target overlap' (Crum et al. [26], 2005); this represents the sensitivity of the analysis and is a measure of segment concordance when there are some areas in the reference segment not fully represented in the target segment

(2) Manually delineated regions vs. individual macroanatomical atlas and vs. age-based template-transformed atlas Overlap between manually delineated regions and regions resulting from the majority vote approach to individual macroanatomical atlas creation or regions coming from age-appropriate average atlas with linear/nonlinear registration/transformation to participant space

MCBI and NIHPD:

$\mathrm{n}=4$ at $3,6,9,12$ months

$\mathrm{n}=2$ at 4.5 and 7.5 months

3) Individual macroanatomical atlases vs. registration/transformation to average macroanatomical atlas Overlap between regions resulting from the majority vote approach to individual MCBI and NIHPD:

$\mathrm{n}=4$ at $3,6,9$, and 12 months $\mathrm{n}=2$ at 4.5 and 7.5 months macro-anatomical atlas creation and linear/nonlinear registration/transformation of an age-based atlas into the participant space

Internal: MCBI

$\mathrm{n}=14,12,14,11,8$, and 10

at $3,4.5,6.0,7.5,9$, and 12

months

External: NIH

$\mathrm{n}=20,32,28$, and 25

at $3,6,9$, and 12 months

source $\left(\mathrm{F}_{1,15}=26.46, \mathrm{p}=0.0014\right)$; similar patterns were present in the means to those described for the Hammers data.

There was a 3-way interaction between the registration type, atlas source and segment, for both Hammers and LPBA40 atlases $\left(\mathrm{F}_{4,56}=14.07, \mathrm{p}<0.0001\right.$ and $\mathrm{F}_{3,42}=6.77$, $\mathrm{p}<0.0001$, respectively). Figure 7 shows the mean Dice values for the Hammers and LPBA40 atlases for the 5 segments for the linear and nonlinear registrations, separately for the participant atlas and the average-transformed atlas. The interaction was due to the size of significant effects rather than the specific pattern. For both the transformed age-appropriate average atlas and the participant atlas, the nonlinear Dice coefficient was larger than the linear Dice coefficient for both the brainstem and thalamus segments. This difference between nonlinear and linear coefficients also occurred for the participant atlas source for the cerebellum segment. Otherwise, all the other segments did not have significantly different Dice coefficients for linear and nonlinear registration methods.

\section{Individual Macroanatomical Atlases and Registration/ Transformation to Average Macroanatomical Atlas (Comparison 3)}

The final analysis examined the overlap between the Hammers or LPBA 40 atlases that were constructed on the 


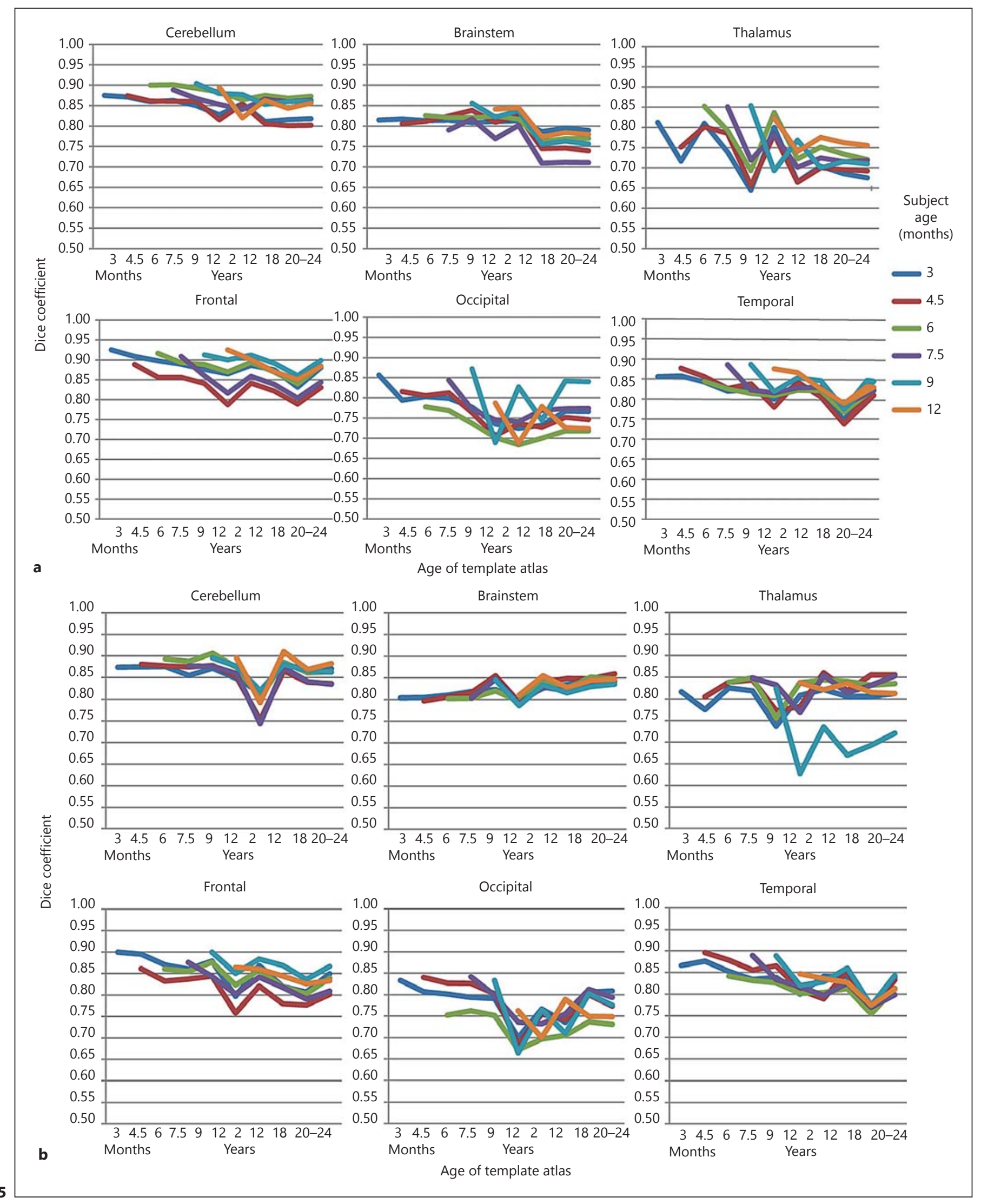

(For legend see next page.) 
individual, with the segments coming from a registration/ transformation from the average atlas to the participant MRI volume space. These atlases were constructed for all infant MRIs. The analysis came from the participants who contributed to the creation of the average atlas to verify the internal validity of this approach. The analysis also used the MRIs from the NIHPD infants who were not included in the creation of the averages, as an external validation of the usefulness of the average atlas. A Dice coefficient was calculated between the average-transformed and the participant atlas by defining the intersection as the agreement for each voxel over all segments of the macroanatomical atlas, thus providing a measure of overall fit between the two atlases.

The multiple-segment Dice coefficient was analyzed with an age $(6$ ages $) \times$ group $(2$ groups: $\mathrm{MCBI}, \mathrm{NIH}) \times$ registration type (2 types) ANOVA, separately for the Hammers and LPBA40 atlases. Figure 8 shows this overall comparison for the Hammers and LPBA40 atlases, separately for the linear and nonlinear methods and the 2 groups. It can be seen in this figure that the fit between the transformed and participant atlases was similar for the 2 groups and was generally about 0.90 . There was a significant main effect for the registration type $\left(\mathrm{F}_{1,165}=\right.$ $165.14, \mathrm{p}<0.0001)$, and the testing group factor was close to significant $\left(\mathrm{F}_{1,165}=3.16, \mathrm{p}=0.0736\right)$. The Dice coefficient for the MCBI infants was larger than the NIH infants (mean $=0.937$ and $0.899, \mathrm{SE}=0.0017$ and 0.0012 , respectively) and the linear methods resulted in a larger Dice coefficient than the nonlinear method (mean = 0.929 and $0.898, \mathrm{SE}=0.0084$ and 0.0077 , respectively). There were no other main effects or interactions.

We also calculated the 'target overlap' [25] for the two atlases. The target overlap represents the intersection of a target and reference overlap divided by the reference volume area. This represents the sensitivity of the analysis and is a measure of segment concordance when there are some areas in the reference segment not fully represented in the target segment. Figure 8 shows the target overlap for the age-transformed and the participant volumes calculated over all the segments. The target overlap for both MCBI and NIH participants was about 0.95 , indicating a very close fit between the average age-transformed and individual participant atlases. An age $(2) \times$ group $(2) \times$ registration type (2) ANOVA showed only a significant effect for the registration type factor; the target overlap for the 2 groups was not significantly different. Finally, we also calculated the target overlap for the 83 areas of the Hammers atlas and the 56 areas of the LPBA 40 atlas. Figure 9 shows the target overlap separately for the MCBI

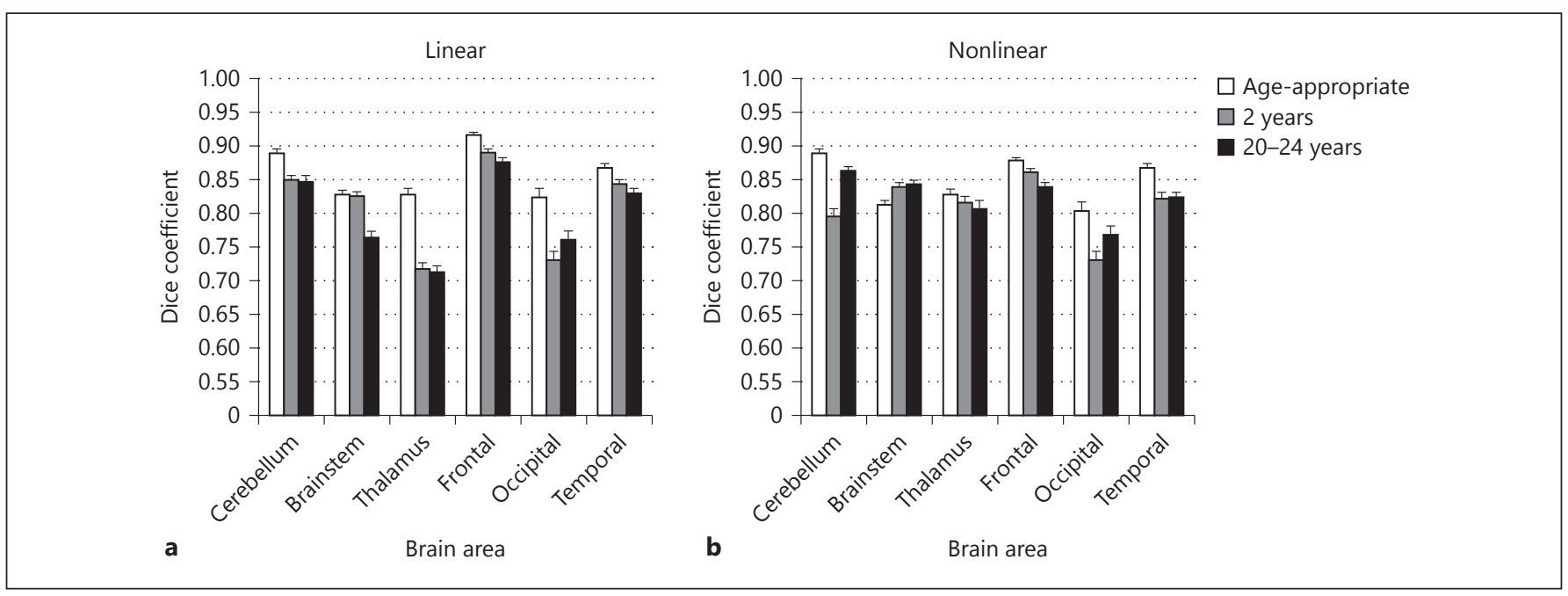

Fig. 6. Overlap between manually delineated regions and regions resulting from registration/transformation to template-based atlases. Graph shows mean Dice value as a function of the brain re-

Fig. 5. Overlap between manually delineated regions and regions resulting from registration/transformation to template-based atlases. Graph shows mean Dice value as a function of the age of the template-based atlas separately for each age group. Each line rep- gion with a bar for each age of the template-based atlas (same age as participant, 2 years, 20-24 years). a Linear. b Nonlinear. Error bars represent $\mathrm{SE}$.

resents a different age group. a Results from linear transformations. b Results from nonlinear transformations. Each subpanel shows the results for 1 of 6 different brain regions. 


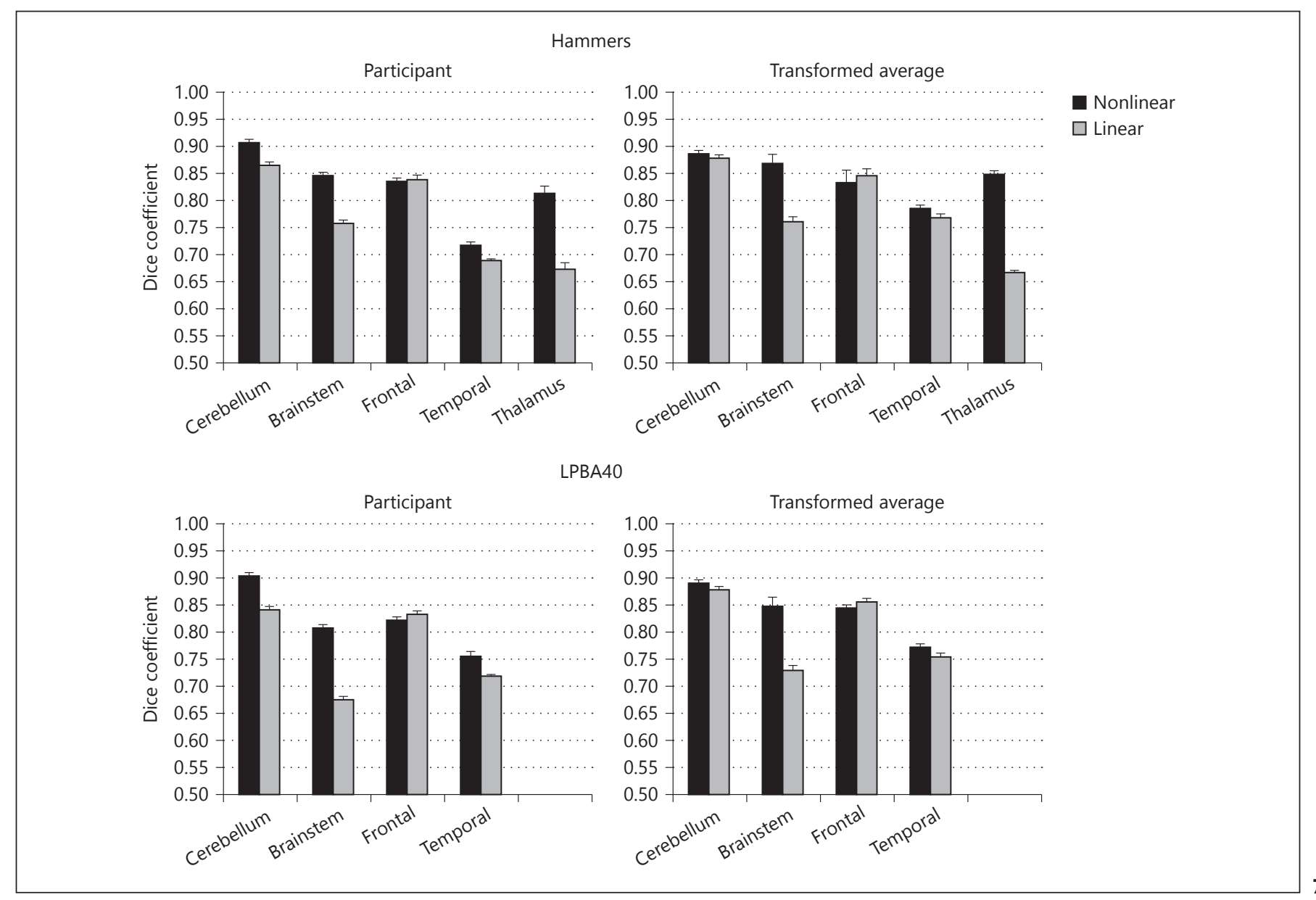

Fig. 7. Overlap between manually delineated regions and Hammers and LPBA40 atlas regions resulting from the majority vote approach to individual participant macroanatomical atlas creation (left) and the ageappropriate average MRI template atlastransformed to the participant (right). Graph shows mean Dice value as a function of the brain region and linear/nonlinear registration. Error bars represent SE.

Fig. 8. Comparison of MCBI (internal validation) and NIHPD (external validation) fit between the participant atlas and ageappropriate average-transformed atlas. The Dice coefficient represents the agreement between multiple segments over all the segments of the atlas and the target overlap is the overlap of the age-appropriate average-transformed atlas to the participant atlas. The SE for each graph was $<0.005$.

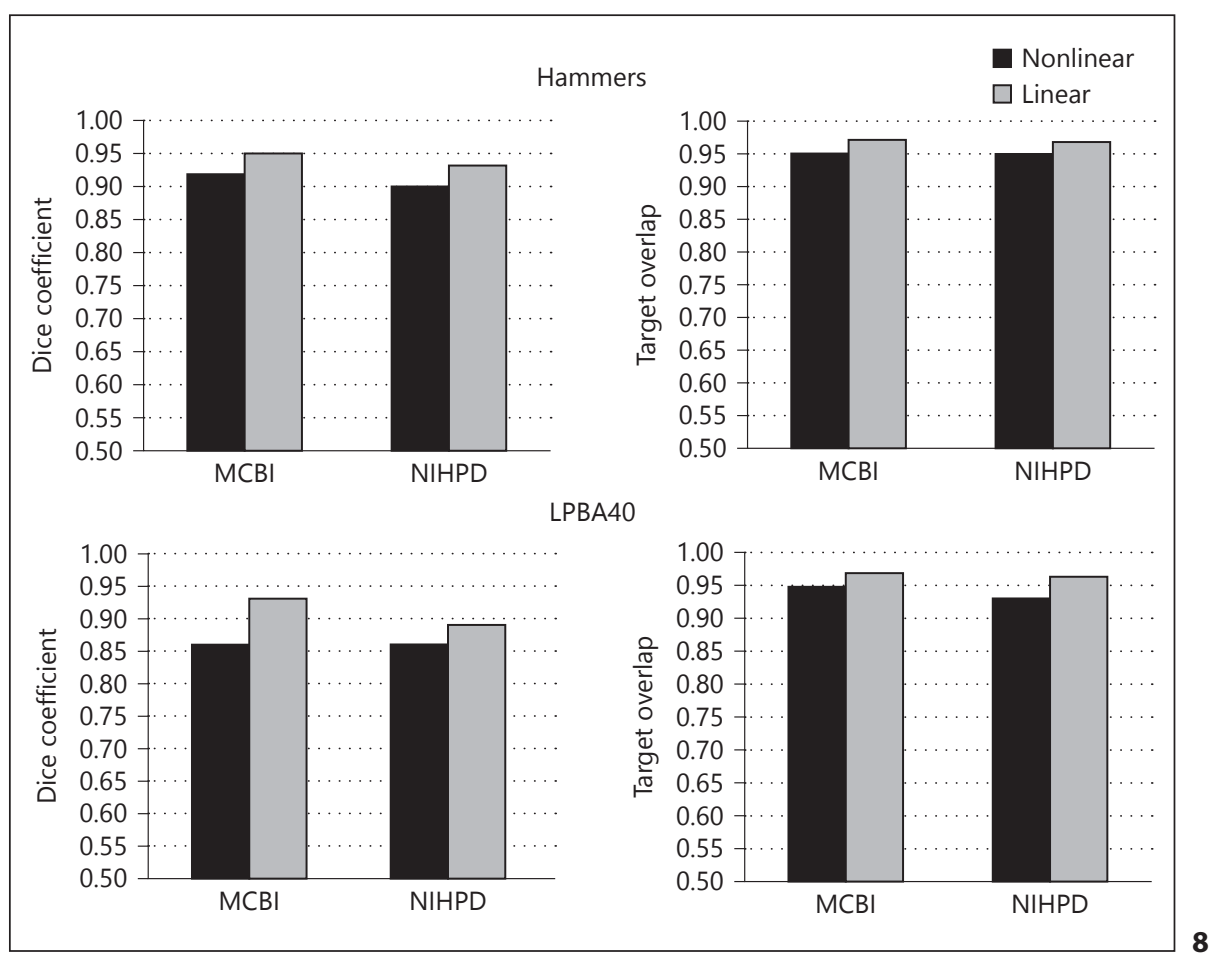




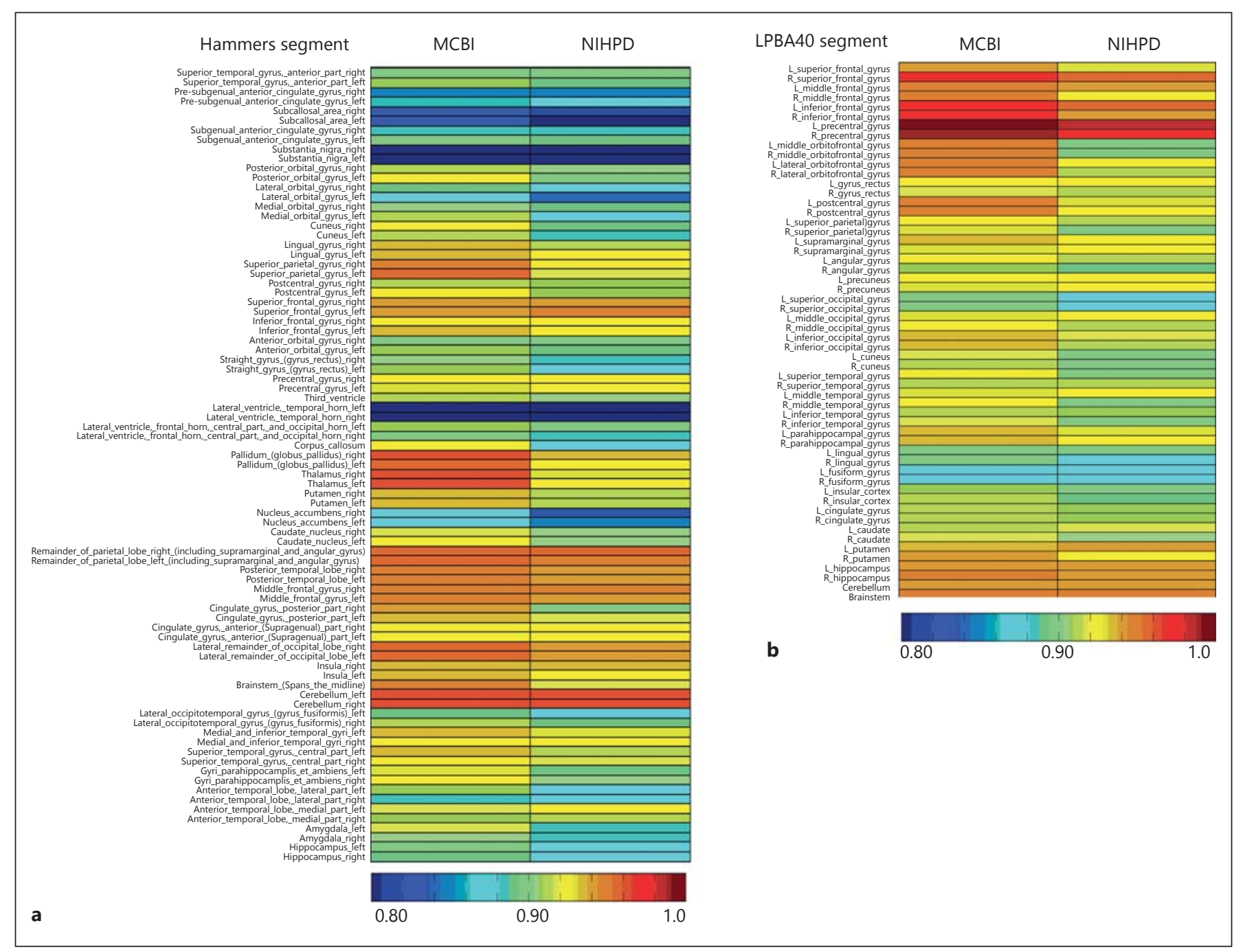

Fig. 9. Target overlap between the participant and the age-appropriate average-transformed atlas, separately for the MCBI (interval validation) and NIHPD (external validation) groups. Each row

and NIHPD groups for the linear registration/transformation and for all the segmented areas of these atlases. There was a good correspondence between the MCBI and the NIHPD groups on the pattern of target overlap across the different segments for both the Hammers and the LPBA40 atlases.

\section{Discussion}

Previous neuroanatomical stereotaxic atlas work in infants has focused either on newborns [26-28] or a few limited ages $[7,11]$. The current work provides viable alternatives for stereotaxic atlas work with infants from 3 to represents 1 segment and the color values represent the target overlap. a Data from the Hammers atlas. b Data from the LPBA40 atlas.

12 months of age with fine-grained ages. We created agespecific stereotaxic atlases for average MRI templates for infants. This was done by manual delineation of average MRI templates [5]. Transforming the average atlas to individual participant MRI space and comparing the overlap of the transformed atlas to manual delineated regions on individual participants tested the validity of the template-based atlases. The overall overlap between the transformed atlas regions and the manually delineated regions was very good (Dice values $\sim 0.85$ ). The largest overlap was with the delineated regions and an atlas from an age-appropriate average MRI template. There was a decreasing overlap between the manually delineated regions and the template-based atlas regions as the age dif- 
ference between the participant and the average atlas increased. Using an age-appropriate template-based atlas provided a significantly higher overlap than using an adult template-based atlas. Overall, these results suggest that automatic atlas segmentation is best done using ageappropriate stereotaxic atlases. The overlap values found with the manually delineated atlases and the internal/external validation comparisons are comparable to work done with adult anatomical atlases based on similar procedures $[2,11]$. We thus expect that our atlases should perform as well as these adult atlases.

A second objective of the current work was to use the procedures of Gousias et al. [11] to create individual macroanatomical atlases for infants from 3 to 12 months. This approach worked similarly for infants across the first year, as well as for the Hammers atlas and the LPBA40 atlas. This procedure should be useful for automatic computation of individual macroanatomical atlases for infant participants. It is possible that a direct registration/transformation from the average to an individual may be sufficient. The overlap between the individual macroanatomical atlases and registration/transformation to an ageappropriate average macroanatomical atlas did not change substantially over age. The values of the Dice coefficients were around 0.90 and the overlap values were near 0.95 . These results were similar when tested with an external validation group. The computational requirements of the procedure of Gousias et al. [11] are quite high, so the use of the average anatomical atlas for automatic computation may suffice for some applications.

There were differences in fit across anatomical regions. For example, the cerebellum and frontal lobes showed better overall fits for the manual atlas compared with the thalamus, occipital lobe and temporal lobe (fig. 6, 7). The areas with lower overlap were also more strongly affected by the use of the atlas from the age-inappropriate MRI template (e.g. occipital lobe, thalamus; fig. 5, 6). Additionally, the occipital lobe region had small Dice values for all participants with the child and adult average templates, whereas the other regions showed larger Dice values for the older infants and these older templates (fig. 5). The nonlinear registration improved the fit between the participant manually delineated segments and the ageappropriate MRI template segments, as well as segments from older ages (fig. 6). These results may be helpful in determining whether to use automatic atlas procedures or manual delineation. For some regions (e.g. frontal lobe, cerebellum) the automatic segmentation may provide results comparable to manual delineation. For others, only manual delineation of segmented areas will pro- vide adequate identification of brain areas. The nonlinear registration improved the fit between the individual participants and either the age-appropriate manual segments or the age-appropriate macroanatomical atlases, particularly for those areas that had lower Dice values from the linear registration.

Some of our comparisons may be described as internal validations of the procedures, whereas others are external tests of the atlases. For example, our comparison of the manually delineated regions against the registration/ transformation to the template atlas (table 4 , Comparison 1) used 4 participants at $3,6,9,12$, and 24 months -2 of these participants also were used to construct the average MRI template upon which the manual delineations were made (MCBI participants) and 2 were not used in the construction of the template (NIHPD participants). We found similar results for the internal validation of the MCBI participants against their age-appropriate atlas and the external validation of the NIHPD participants against their age-appropriate atlas. Both the internal and external validation participants showed a closer fit to the age-appropriate atlas than to the atlases based on older groups (e.g. 2-year-old or adult atlas; fig. 5, 6). Similarly, the comparison of the majority vote fusion age-appropriate average atlas with the registration/transformed atlas was done both with the MCBI participants (table 4, comparison 3 , internal) and NIHPD participants (table 4, comparison 3 , external). The results from the internal validation comparisons were nearly identical in the pattern of the results with the external validation comparisons, with slightly higher Dice coefficients for the internal validation (fig. 8). Also, the pattern of overlap across the individual segments of the Hammers and LPBA40 atlases was very similar for the MCBI and the NIHPD groups (fig. 9). An important note for the external validation sample is that it was composed entirely of 1.5-tesla, 2D, $1 \times 1 \times 3 \mathrm{~mm}$ scans. These scans have poorer spatial resolution due to the smaller magnet strength, the $2 \mathrm{D}$ sampling and the thicker slices. The similar performance of the validations with the external and internal validation comparisons implies these atlases will generalize to a wide range of scanning protocols. The slightly smaller Dice coefficients for the external validation comparisons probably represent the level to which these results will be generalized to a new sample of MRIs, though we expect that MRIs with the same high-resolution characteristics as the MCBI sample may work better than the lower-resolution NIHPD MRI volumes.

These results highlight some of the limitations and advantages of different atlas approaches as they apply to in- 
fants. Gilmore et al. [26] and Gousias et al. [27] developed procedures for delineating structures on each individual newborn brain (approach 4 in table 1). Depending on the region of interest, automatically registering an infant to an age-appropriate atlas (approach 2) may work well enough to eliminate the need for time-consuming individual delineation. The current work shows that automatic linear registration of a 1-year-old's brain to a 2-year-old atlas (approach 2 [7] or 6-month-old to Montreal Neurological Institute, MNI, atlas [6]) does not work as well as registration to an age-appropriate atlas, and there were several places where nonlinear registration improved the results. If one desires a macroanatomical atlas, one could use a majority vote approach (approach 3 [11]) to create individual macroanatomical atlases for infant participants without needing manual delineation. Additionally, this could be done for child or adolescent groups [29] or even extended to studies of development in subcortical brain areas [30]. However, this procedure is resource intensive. Alternatively, one could register/ transform the infant participants to an age-appropriate average macroanatomical atlas, with similar precision across ages.

Our choice of 1.5-month atlas intervals across the range from 3 to 9 months resulted in about 8-14 infants for the average template at each age [10]. We do not know whether these numbers of participants in the average template resulted in less reliable templates or anatomical atlases for each age. However, we prefer the approach of having smaller age ranges for our averages given the tremendous development in brain structure across the first year of life. We feel that the smaller the age range for the templates and atlases the more likely it is that age-appropriate atlases will enhance both research and clinical use of these atlases. The overlap values found with both the internal and external validation procedures are comparable to work done with adult anatomical atlases based on similar procedures, so we expect that our atlases should perform as well as adult work. However, it could be preferable to obtain a much larger set of infant MRIs, perhaps at the same age, to examine the effect of sample size on the reliability of the anatomical atlases. These cautions apply equally to the age-appropriate average templates that exist for infant ages (with 6-montholds [6], with 2-year-olds [11], with these ages [5], and with newborn, 12-month-olds and 24-month-olds [7]).

\section{Acknowledgments}

This work was supported by NIH grant R37 HD18942 to J.E.R.

Data used in the preparation of this article were obtained from the NIH Pediatric MRI Data Repository created by the NIH MRI Study of Normal Brain Development. This is a multisite, longitudinal study of typically developing children from ages newborn through young adulthood conducted by the Brain Development Cooperative Group and supported by the National Institute of Child Health and Human Development, the National Institute on Drug Abuse, the National Institute of Mental Health, and the National Institute of Neurological Disorders and Stroke (Contract No. N01-HD02-3343, N01-MH9-0002 and N01-NS-9-2314, $-2315,-2316,-2317,-2319$, and -2320$)$. A listing of the participating sites and a complete listing of the study investigators can be found at http://www.bic.mni.mcgill.ca/nihpd/info/participating centers.html.

The adult brain atlases from www.brain-development.org were used in the preparation of this article (copyright Imperial College of Science, Technology and Medicine, 2007, all rights reserved).

The authors would like to acknowledge Kristen Sloan and Meagan Bang for their help with manual delineations.

\section{References}

1 Richards JE, Xie W: Brains for all the ages: structural neurodevelopment in infants and children from a life-span perspective; in Bensen J (ed): Advances in Child Development and Behavior. Elsevier, Amsterdam, 2015.

$\checkmark 2$ Shattuck DW, et al: Construction of a 3D probabilistic atlas of human cortical structures. Neuroimage 2008;39:1064-1080.

-3 Altaye M, et al: Infant brain probability templates for MRI segmentation and normalization. Neuroimage 2008;43:721-730.

4 Kazemi K, et al: A neonatal atlas template for spatial normalization of whole-brain magnetic resonance images of newborns: preliminary results. Neuroimage 2007;37:463-473.

5 Sanchez CE, et al: Neurodevelopmental MRI brain templates for children from 2 weeks to 4 years of age. Dev Psychobiol 2011;54:77-91.
6 Akiyama LF, et al: Age-specific average head template for typically developing 6-monthold infants. PLoS One 2013;8:e73821.

7 Shi F, et al: Infant brain atlases from neonates to 1- and 2-year-olds. PLoS One 2011; 6:e18746.

8 Tzourio-Mazoyer N, et al: Automated anatomical labeling of activations in SPM using a macroscopic anatomical parcellation of the MNI MRI single-subject brain. Neuroimage 2002;15:273-289.

-9 Sanchez CE, et al: Age-specific MRI templates for pediatric neuroimaging. Dev Neuropsychol 2012;37:379-399.

10 Richards JE, et al: A database of age-appropriate average MRI templates. Neuroimage 2015, DOI: 10.1016/j.neuroimage.2015.04. 055 .
1 Gousias IS, et al: Automatic segmentation of brain MRIs of 2-year-olds into 83 regions of interest. Neuroimage 2008;40:672-684.

12 Hammers A, et al: Three-dimensional maximum probability atlas of the human brain, with particular reference to the temporal lobe. Hum Brain Mapp 2003;19:224-247.

$\checkmark 13$ Heckemann RA, et al: Automatic anatomical brain MRI segmentation combining label propagation and decision fusion. Neuroimage 2006;33:115-126.

14 Song J, et al: Anatomically accurate head models and their derivatives for dense array EEG source localization. Funct Neurol Rehabil Ergon 2013;3:275-293.

15 Yoon U, et al: The effect of template choice on morphometric analysis of pediatric brain data. Neuroimage 2009;45:769-777. 
16 Fonov V, et al: Unbiased average age-appropriate atlases for pediatric studies. Neuroimage 2011;54:313-327.

17 Sanchez CE, et al: Neurodevelopmental MRI brain templates for children from 2 weeks to 4 years of age. Dev Psychobiol 2012;54:77-91.

18 Avants BB, et al: Symmetric diffeomorphic image registration with cross-correlation: evaluating automated labeling of elderly and neurodegenerative brain. Med Image Anal 2008;12:26-41.

19 Klein A, et al: Evaluation of 14 nonlinear deformation algorithms applied to human brain MRI registration. Neuroimage 2009;46:786802.

20 Almli CR, et al: The NIH MRI study of normal brain development (Objective-2): newborns, infants, toddlers, and preschoolers. Neuroimage 2007;35:308-325.

21 Leppert IR, et al: T2 relaxometry of normal pediatric brain development. J Magn Reson Imaging 2009;29:258-267.

22 Heckemann RA, et al: Automatic anatomical brain MRI segmentation combining label propagation and decision fusion. Neuroimage 2006;33:115-126.

23 Heckemann RA, et al: Information extraction from medical images (IXI): developing an eScience application based on the Globus Toolkit. Proceedings 2nd UK e-Science AllHands Conference, Nottingham, 2003.

24 Jenkinson M, Smith S: A global optimisation method for robust affine registration of brain images. Med Image Anal 2001;5:143-156.

25 Crum WR, et al: Generalised overlap measures for assessment of pairwise and groupwise image registration and segmentation. Med Image Comput Comput Assist Interv 2005;8:99-106.

26 Gilmore $\mathrm{JH}$, et al: Regional gray matter growth, sexual dimorphism, and cerebral asymmetry in the neonatal brain. J Neurosci 2007;27:1255-1260.

27 Gousias IS, et al: Magnetic resonance imaging of the newborn brain: manual segmentation of labelled atlases in term-born and preterm infants. Neuroimage 2012;62:1499-1509.

28 Oishi K, et al: Multi-contrast human neonatal brain atlas: application to normal neonate de- velopment analysis. Neuroimage 2011;56:820.

29 Hashimoto T, et al: Increased posterior hippocampal volumes in children with lower increase in body mass index: a 3-year longitudinal MRI study. Dev Neurosci 2015;37:153-160.

30 Uda S, et al: Normal development of human brain white matter from infancy to early adulthood: a diffusion tensor imaging study. Dev Neurosci 2015;37:182-194.

31 Talairach J, Tournoux P: Co-Planar Stereotaxic Atlas of the Human Brain. Stuttgart, Thieme, 1988.

32 Mazziota J,et al: A probabilistic atlas and reference system for the human brain: International Consortium for Brain Mapping (ICBM). Philos Trans R Soc Lond B 2001;356:1293-1322.

33 Desikan RS, et al: An automated labeling system for subdividing the human cerebral cortex on MRI scans into gyral based regions of interest. Neuroimage 2006;31:968-980.

34 Dice LR: Measures of the amount of ecologic association between species. Ecology 1945;26: 297-302. 